\title{
Controlling potential landscape of heterostructured nanocrystals with interfacial polarization
}

\section{Byeong Guk Jeong}

Sungkyunkwan University

Jun Hyuk Chang

Sungkyunkwan University

Donghyo Hahm

Sungkyunkwan University

\section{Seunghyun Rhee}

Sungkyunkwan University

Myeongjin Park

Samsung Display

\section{Sohoo Lee}

Samsung Display

Youngdu Kim

Sungkyunkwan University

Doyoon Shin

Sungkyunkwan University

Jeong Woo Park

Sungkyunkwan University

Changhee Lee

Seoul National University https://orcid.org/0000-0003-2800-8250

Doh C. Lee

KAIST https://orcid.org/0000-0002-3489-6189

Kyoungwon Park

Korea Electronics Technology Institute

\section{Euyheon Hwang}

Sungkyunkwan University

Wan Ki Bae ( $\nabla$ wkbae@skku.edu )

Sungkyunkwan University

Article 
Keywords: h-NC solids, Steering Dipole Moments, Vacuum-level Shift, Photochemical Stability, Optoelectronic Performance

Posted Date: December 10th, 2020

DOI: https://doi.org/10.21203/rs.3.rs-120373/v1

License: (1) This work is licensed under a Creative Commons Attribution 4.0 International License. Read Full License

Version of Record: A version of this preprint was published at Nature Materials on November 18th, 2021. See the published version at https://doi.org/10.1038/s41563-021-01119-8. 


\section{Controlling potential landscape of heterostructured}

\section{nanocrystals with interfacial polarization}

Byeong Guk Jeong ${ }^{1} \uparrow$, Jun Hyuk Chang ${ }^{1} \uparrow$, Donghyo Hahm ${ }^{1} \uparrow$, Seunghyun Rhee ${ }^{1}$, Myeongjin Park $^{2}$, Sooho Lee ${ }^{2}$, Youngdu Kim ${ }^{1}$, Doyoon Shin ${ }^{1}$, Jeong Woo Park ${ }^{1}$, Changhee Lee ${ }^{2}$, Doh C.

Lee $^{3 *}$, Kyoungwon Park ${ }^{4}$, Euyheon Hwang ${ }^{1 *}$, and Wan Ki Bae ${ }^{1 *}$

${ }^{1}$ SKKU Advanced Institute of Nanotechnology and Department of Nano Engineering, Sungkyunkwan University, Suwon 16419, Republic of Korea.

${ }^{2}$ Samsung Display Research Center, Samsung Display, Yongin 17113, Republic of Korea.

${ }^{3}$ Department of Chemical and Biomolecular Engineering, KAIST Institute for the Nanocentury, Korea Advanced Institute of Science and Technology (KAIST), Daejeon 34141, Republic of Korea.

${ }^{4}$ Display Research Center, Korea Electronics Technology Institute (KETI), Seongnam 13509, Republic of Korea.

\section{Corresponding Authors}

Correspondence to Wan Ki Bae (wkbae@skku.edu); Euyheon Hwang (euyheon@skku.edu);

Doh C. Lee (clee@,kaist.edu) 
Abstract: The potential profile and the energy level offset of core/shell heterostructured nanocrystals ( $h$-NCs) determine the photophysical properties and the charge transport characteristics of $h$-NC solids. However, limited material choices for heavy metal-free III-V/IIVI $h$-NCs pose challenges in comprehensive control of the potential profile. Herein, we present an unprecedented approach to such control by steering dipole moments at the interface of IIIV/II-VI $h$-NCs. The controllable heterovalency at the interface is responsible for interfacial dipole moments that result in the vacuum-level shift, providing an additional knob for the control of optical and electrical characteristics of $h$-NCs. We capitalize on the atomic precision with which to synthesize $h$-NCs by correlating interfacial dipole moments to photochemical stability and optoelectronic performance of resulting $h$-NCs. 
Colloidal semiconductor nanocrystals (NCs) exhibit size-dependent tunable bandgap and narrow emission bandwidth ${ }^{1-5}$, which put the materials on the map in optoelectronic ${ }^{6-12}$ and biological applications ${ }^{13-16}$. Advances in the wet-chemical synthesis have created a fertile opportunity space for NCs to contribute to the progress in those areas. The potential profile in heterostructured nanocrystals ( $h$-NCs) gives rise to the spatial control of electron and hole wavefunctions and their overlap integral, which determine photophysical and photochemical characteristics of the $h$-NCs ${ }^{10,17-19}$. For example, in the type I core/shell heterostructure, bandedge energy levels are offset in a straddled configuration, making both electron and hole are confined within the emissive core surrounded by the shell of a greater bandgap ${ }^{10,17,20}$. The increased overlap of electron and hole wavefunctions at the core results in high emission efficiency with prolonged stability.

The change of the potential profile in $h$-NCs has profound bearing on the electron- and hole-injection into the $h$-NCs from adjacent charge transport layers. The early rapid progress on $h$-NC-based light-emitting diodes was built on the relative ease, with which II-VI/II-VI core/shell heterostructures (e.g., $\mathrm{CdSe} / \mathrm{CdZnS}$ or $\mathrm{ZnSe} h$-NCs) are prepared with varying their potential profiles by control of their chemical compositions ${ }^{21-23}$. With the environmental ramifications on the table, the use of $h$-NCs free of toxic elements casts undeniable importance. One prominent example highlights III-V/II-VI $h$-NCs (e.g., InP/ZnSe or ZnS $h$-NCs) ${ }^{20,24-26}$. Notwithstanding the rapid progress in InP-based $h$-NCs for light-emitting devices, the limited choice for the compositions of III-V/II-VI $h$-NCs poses a roadblock to exquisite control of potential profile in $h$-NCs, ultimately undermining the practical use of III-V/II-VI $h$-NCs ${ }^{27,28}$.

Here, we introduce a universal scheme that helps fine tuning of the potential profile of III-V/II-VI $h$-NCs: controlling dipole moments arising from the heterovalency at the interface 
between III-V core and II-VI shell. First, we examine the role of heterovalent bonds (III-VI and II-V bonds) at the interface of III-V/II-VI $h$-NCs as the source of interfacial electric dipoles that modulate the potential profile of $h$-NCs and the electronic energy levels. The cation exchange process at III-V/II-VI heterovalent interface results in the change in the interfacial polarization. Finally, we discuss the impact of engineered interfacial dipoles on the transport characteristics of charges across $h$-NC and surrounding media and thus on the performance of end-applications utilizing optical and optoelectronic properties of III-V/II-VI $h$-NCs.
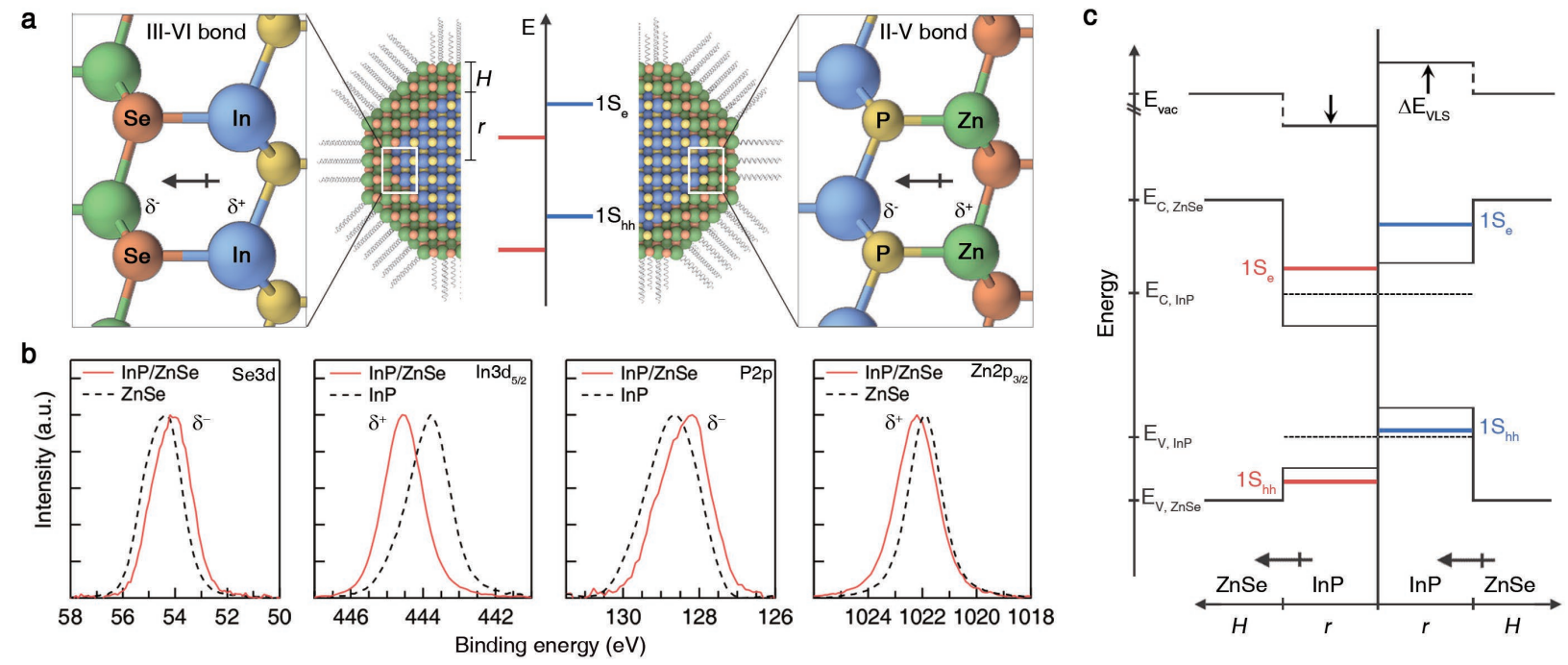

Fig. 1. Formation of bond dipoles at the InP/ZnSe interface and their impact on the potential profile of $\boldsymbol{h}$-NCs. (a) Schematics illustrating bond dipoles at the (111) interface of zinc blende InP/ZnSe $h$-NCs. The In-Se (left) and Zn-P (right) bonds create outward and inward dipole moments, respectively. The interfacial dipole moments accompany the change in the electronic energy levels of $h$-NCs. (b) X-ray photoemission spectra (XPS) of Se3d, In3d5/2, P2p, and $\mathrm{Zn} 2 \mathrm{p}_{3 / 2}$ obtained from InP/ZnSe $h$-NCs (red solid lines). XPS spectra of $\operatorname{In} 3 \mathrm{~d}_{5 / 2}$ and P2p obtained from InP NCs and Se3d and Zn2p $3 / 2$ obtained from ZnSe NCs are overlayed for comparison (black dashed lines). (c) Schematic illustration of the change in the potential profile 
of $\mathrm{InP} / \mathrm{ZnSe} h$-NCs in the presence of outward (left) versus inward (right) dipole moments at the heterovalent interface. The dipole moments at the interface cause the vacuum-level shift that alters the electronic energy levels. The lowest quantized states of conduction band $\left(1 \mathrm{~S}_{\mathrm{e}}\right)$ and valance band $\left(1 \mathrm{~S}_{\mathrm{hh}}\right)$ are depicted with colored lines.

There are two types of heterovalent bonds (III-VI and II-V bonds) that construct dipole moments at the interface of III-V/II-VI $h$-NCs. Fig. 1 exemplifies the case of zinc blende (ZB) InP/ZnSe core/shell $h$-NCs. X-ray photoelectron spectroscopy (XPS) reveals that the In-Se bonding at the interface increases the binding energy of $\operatorname{In} 3 \mathrm{~d}_{5 / 2}$ but decreases the binding energy of Se3d5/2 (Fig. 1b, Supplementary Fig. 1), reciting the obvious notion that indium is partially positively charged $\left(\delta^{+}\right)$and selenium is partially negatively charged $\left(\delta^{-}\right)$to create a bond dipole moment pointing from In to Se. In the same manner, Zn-P bonding yields a bond dipole moment pointing from Zn to P (Fig. 1b, Supplementary Fig. 1). The vector sum of bond dipole moments from In-Se bonds and $\mathrm{Zn}-\mathrm{P}$ bonds constructs the net dipole moments at the interfaces. For instance, the dipole moments materialize at the polar planes (e.g., $\{111\}$ or $\{100\})$ of ZB $\mathrm{InP} / \mathrm{ZnSe} h$-NCs, whereas the dipole moments at the heterojunctions at $\{110\}$ planes remain neutral (see Supplementary Fig. 2 and supplementary Note 1). Supplementary Fig. 2 shows calculation results based on the ab-initio density functional theory, corroborating the net shift of charges at the interface.

The difference of dipole moments produced by In-Se bonds and dipole moments by Zn-P bonds creates a net electric field at the interface ${ }^{29,30}$ that gives rise to the change in the potential profile of InP/ZnSe $h$-NCs (Fig. 1c). In general, the outward dipole moment lowers the potential of InP core, whereas the inward dipole moment elevates it. The vacuum-level shift of the core 
alters the potential profile between the III-V core and the II-VI shell, which consequently redefines the electronic energy level and the spatial distribution of charge carriers.

The magnitude of the vacuum-level shift $\left(\Delta E_{V L S}\right)$ resulting from the interfacial dipole moments is given by

$$
\Delta E_{V L S}=\frac{n \mu_{\perp}}{A \kappa \varepsilon_{0}}
$$

where $n$ is the total number of bond dipole at the interface, $\mu_{\perp}$ is the dipole moments normal to the interface, $A$ is the interfacial area, $\kappa$ is the average dielectric constant of the interfacial dipole layers, and $\varepsilon_{0}$ is permittivity of free space ${ }^{31}$. In $\mathrm{InP} / \mathrm{ZnSe}$ core/shell $h$-NCs, the total number of In-Se and $\mathrm{Zn}-\mathrm{P}$ bonds is determined by the contact area, the atomic In:P ratio in InP core is directly linked to the magnitude and the direction of interfacial dipole moments. Here, we synthesize $\mathrm{InP} / \mathrm{ZnSe} h$-NCs with a fixed dimension $(\mathrm{InP}$ core radius $(r)=1.9 \mathrm{~nm}$ and $\mathrm{ZnSe}$ shell thickness $(H)=2.2 \mathrm{~nm})$ but varying In:P ratios. The interfacial dipole densities $(\rho)$ are calculated to be $1.50,0.44$, and $0.09 \mathrm{~nm}^{-2}$ from the given geometry of $\mathrm{InP} / \mathrm{ZnSe} h$-NCs with $\mathrm{In}: \mathrm{P}$ ratios of $1.12,1.09$, and 1.07, respectively. The interfacial dipole density $(\rho)$ denotes the average areal density of net bond dipoles at the heterovalent interface. The synthesis procedures, sample information, and calculation method of $\rho$ are detailed in Methods and Supplementary Information (Supplementary Fig. 3, Supplementary Tables 1 and 2, and Supplementary Note 2). 

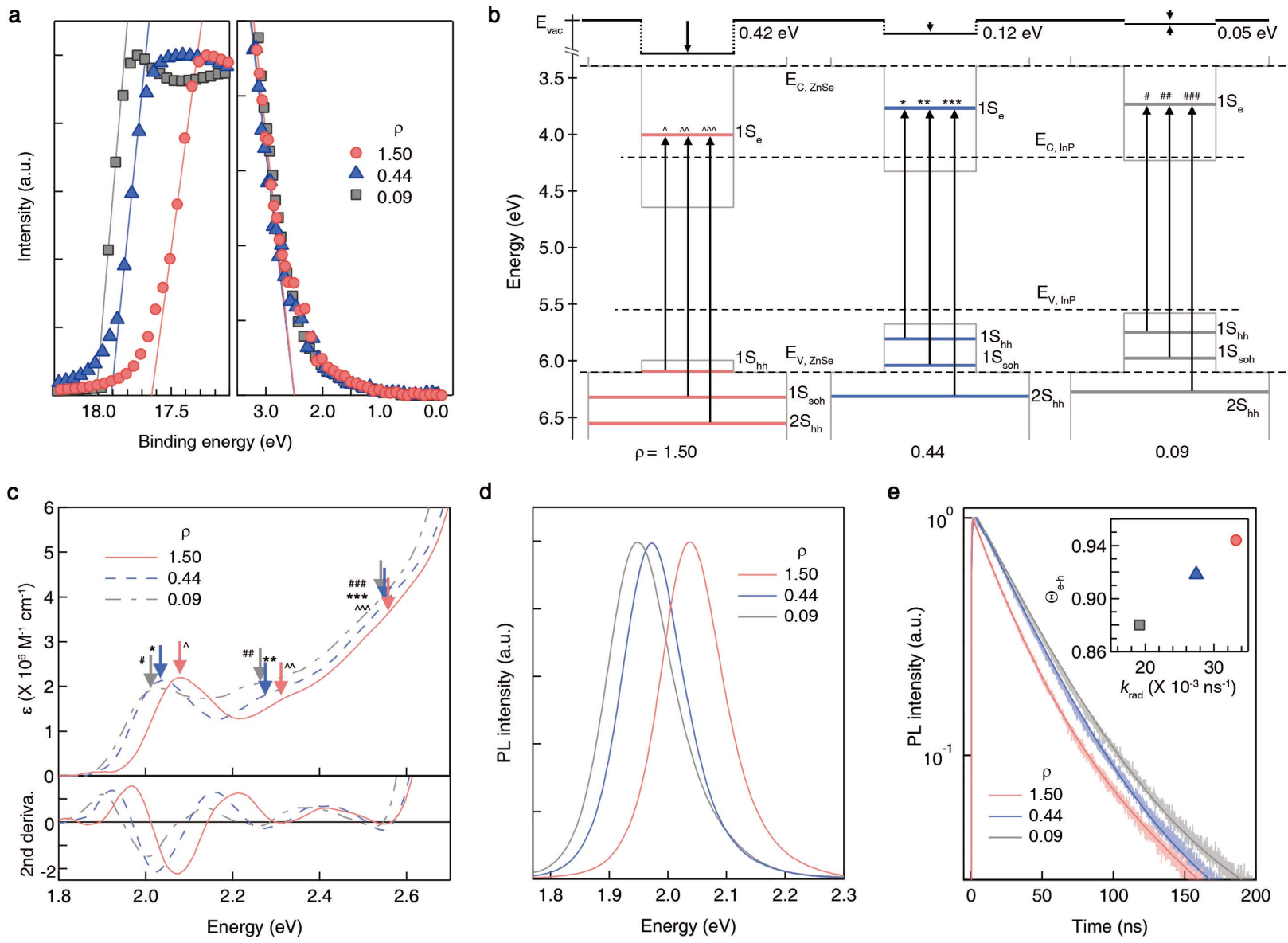

Fig. 2. Electronic and photophysical characteristics of InP/ZnSe $h$-NCs for various In:P

ratios (i.e., for varying interfacial dipole densities, $\rho$ ). (a) Ultraviolet photoemission spectra at the high-binding-energy (left) and the low-binding-energy cutoff region (right) of InP ( $r=1.9$

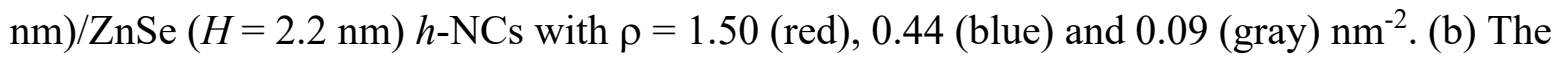
potential profile and the electronic energy levels in $\mathrm{InP}(r=1.9 \mathrm{~nm}) / \mathrm{ZnSe}(H=2.2 \mathrm{~nm}) h$-NCs with $\rho=1.50$ (left), 0.44 (middle) and 0.09 (right) $\mathrm{nm}^{-2}$. Dashed lines denote bulk band edge positions of ZB InP and ZB ZnSe. Dim solid lines indicate the band edge profile of InP/ZnSe $h$ NCs with the interfacial polarization. The lowest quantized state of conduction band $\left(1 \mathrm{~S}_{\mathrm{e}}\right)$ and the quantized states of valence band (1Shh, $1 \mathrm{~S}_{\mathrm{soh}}$, and $\left.2 \mathrm{Shh}_{\mathrm{hh}}\right)$ of $h$-NCs are noted with colored solid lines. Optical transitions ( $1 \mathrm{~S}_{\mathrm{hh}}-1 \mathrm{~S}$ e, $1 \mathrm{~S}_{\mathrm{soh}}-1 \mathrm{~S}_{\mathrm{e}}$, and $\left.2 \mathrm{~S}_{\mathrm{hh}}-1 \mathrm{~S}_{\mathrm{e}}\right)$ at each $h$-NC are indicated with 
arrows and labels. (c) Absorbance spectra (top) and their 2nd derivatives (bottom), (d) photoluminescence spectra, and (e) single exciton decay dynamics (inset: electron-hole wavefunction overlap integral $\left(\Theta_{\mathrm{e}-\mathrm{h}}\right)$ versus radiative recombination rate $\left.\left(k_{\mathrm{rad}}\right)\right)$ of $\operatorname{InP}(r=1.9$ $\mathrm{nm}) / \mathrm{ZnSe}(H=2.2 \mathrm{~nm}) h$-NCs with $\rho=1.50$ (red), 0.44 (blue) and 0.09 (gray) $\mathrm{nm}^{-2}$.

Fig. 2 embodies the impact of interfacial bond dipoles on the electronic and photophysical characteristics of InP/ZnSe $h$-NCs. The ultraviolet photoemission spectroscopy (UPS) shows that the highest quantized state of the valence band (1S of a heavy hole, $1 \mathrm{Shh}$ ) moves down for $\mathrm{InP} / \mathrm{ZnSe} h$-NCs because of the net outward interfacial dipole moments (Fig. 2a). The magnitude of the energy shift in $h$-NCs is proportional to the magnitude of interfacial dipole density ( $\rho)$. Fig. $2 \mathrm{c}$ shows the absorption spectra as a function of energy for various dipole densities. The energy difference of $1 \mathrm{~S}_{\mathrm{hh}}$ within this density range is measured to be $350 \mathrm{meV}$, in close proximity to the vacuum-level shift ( $\left.\Delta E_{V L S}\right)$ of InP core by $370 \mathrm{meV}$ (Fig. 2b). The band offset between the core and the shell alters drastically. Such offset is responsible for the changes in the spatial distribution of charge carriers and their confinement energy in $h$-NCs (Fig. 2c-e). The energy shift of conduction energy levels is more significantly affected by the polarization than that of valence energy levels because of the smaller effective mass of electrons $\left(\mathrm{m}_{\mathrm{e}} / \mathrm{m}_{0}=\right.$ 0.077 vs. $\mathrm{m}_{\mathrm{h}} / \mathrm{m}_{0}=0.56$ for $\left.\mathrm{InP}\right)^{25}$. For example, the electron confinement energy is $70 \mathrm{meV}$ higher for InP/ZnSe $h$-NCs with $\rho=1.50 \mathrm{~nm}^{-2}$ than that with $\rho=0.09 \mathrm{~nm}^{-2}$, whereas the difference of hole confinement energy is relatively small $(20 \mathrm{meV})$, resulting in the substantial modification in the optical bandgap and photoluminescence among $h$-NCs constructed in the same dimension and geometry (Fig. 2c, d). In addition, due to the small effective mass, the electron wavefunction is more likely to leak into the $\mathrm{ZnSe}$ shell upon the reduction of the 
potential barrier, while the hole wavefunction remains confined within the InP core

(Supplementary Fig. 4). The asymmetric changes of charge carriers result in the reduction of the overlap integral of electron and hole wavefunctions $\left(\Theta_{\mathrm{e}-\mathrm{h}}\right)$ and consequently the decrease in their radiative recombination rates ( $k_{\text {rad }}$ (Fig. 2e). Regardless of the dipole density, InP/ZnSe $h$-NCs show near-unity photoluminescence quantum yields (PL QYs) (>90\%) and retain their photophysical properties for an extended period, implying that the chosen structural variation at the interface does not necessarily impair the structural stability of $h$-NCs.

a

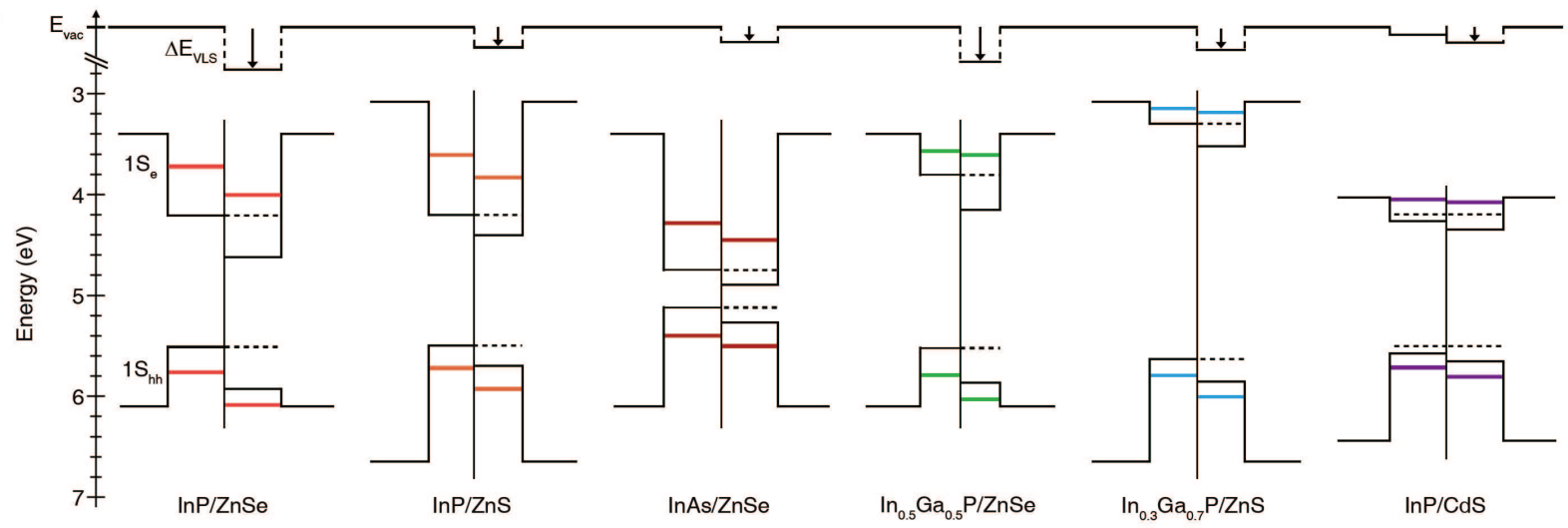

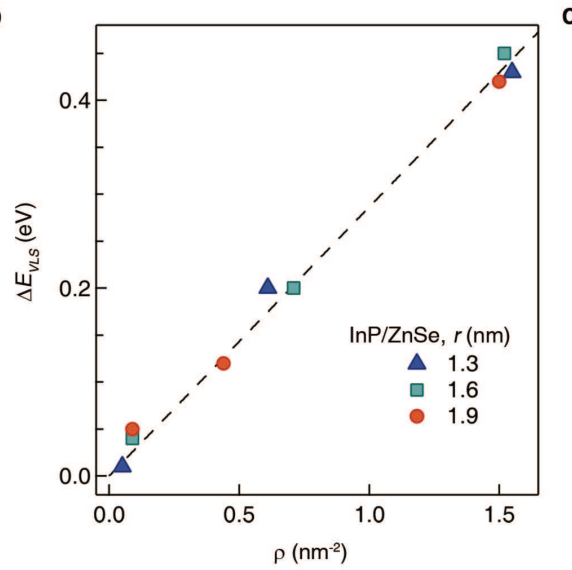

C

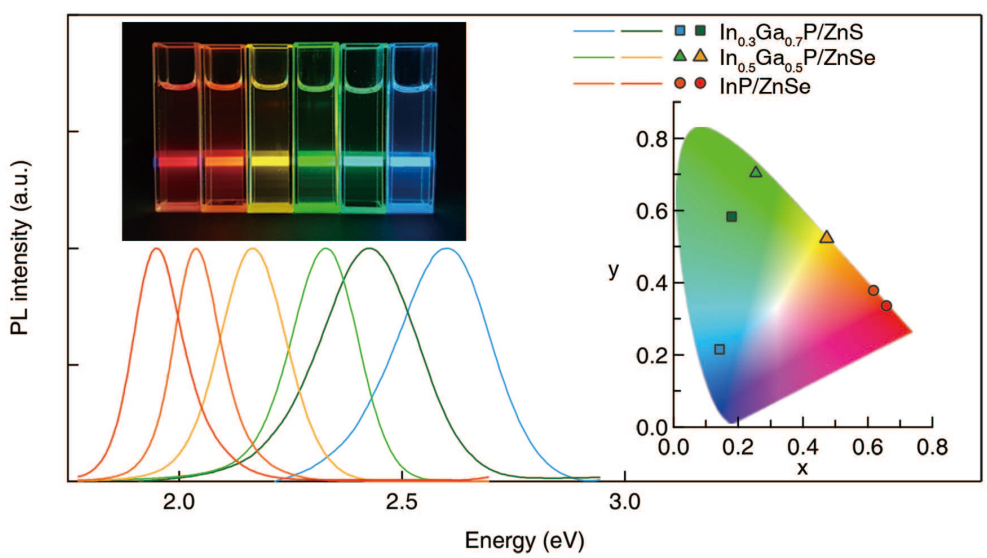

Fig. 3. Universal polarization effect at the interface on electronic and optical characteristics

of III-V/II-VI $\boldsymbol{h}$-NCs. (a) Experimentally gained vacuum-level shift ( $\left.\Delta E_{V L S}\right)$ in InP/ZnSe, InP/ZnS, InAs/ZnSe, In0.5Ga0.5P/ZnSe, In $0.3 \mathrm{Ga} 0.7 \mathrm{P} / \mathrm{ZnS}$, and InP/CdS $h$-NCs upon the change of 
the interfacial polarization. Top solid line indicates the vacuum level ( $\left.E_{\text {vac }}\right)$. The vertical arrows denote experimentally achievable vacuum-level shift with the interfacial polarization for each $h$ NCs. Left and right halves for each $h$-NCs contrast the potential profile of $h$-NCs with the least and highest dipole densities among experimentally achieved values, respectively. The colored solid lines indicate the lowest quantized states of conduction band ( $\left.1 \mathrm{~S}_{\mathrm{e}}\right)$ and valence band (1Shh) for each case. (b) The extent of vacuum-level shift $\left(\Delta E_{V L S}\right)$ versus interfacial dipole density $(\rho)$ in $\mathrm{InP} / \mathrm{ZnSe} h$-NCs of varying InP radii ( $r=1.3$ (blue), 1.6 (green), and 1.9 (red) nm). The $\mathrm{ZnSe}$ thickness $(H)$ is fixed to be $2.2 \mathrm{~nm}$. The dotted line is a linear fit to the data. (c) Achievable photoluminescence spectra from $\operatorname{In}_{0.3} \mathrm{Ga}_{0.7} \mathrm{P}(r=1.2 \mathrm{~nm}) / \mathrm{ZnS}(H=2.6 \mathrm{~nm}), \operatorname{In}_{0.5} \mathrm{Ga} 0.5 \mathrm{P}(r=1.3$ $\mathrm{nm}) / \mathrm{ZnSe}(H=3.2 \mathrm{~nm})$ and $\mathrm{InP}(r=1.9 \mathrm{~nm}) / \mathrm{ZnSe}(H=2.2 \mathrm{~nm}) h$-NCs by varying the interfacial dipole densities. Insets are a photograph of resulting $h$-NCs and their color space in the CIE 1931 chromaticity diagram.

The atomic composition at the heterovalent interface is directly linked to the interfacial dipole density that reshapes the potential profile of III-V/II-VI $h$-NCs. As a mean to control the atomic ratio, we synthesize III-V NCs with group III element-rich surfaces and foster the cation exchange reaction at the surface prior to the passivation of II-VI shells. The introduction of group II precursors chelated with hard bases (e.g., chloride or acetate) in a solution containing III-V NCs results in spontaneous exchange of group III elements with group II elements at the surface based on hard-soft-acid-base principle ${ }^{32,33}$. X-ray diffraction analysis shows that the cation exchange primarily occurs at the polar surfaces (e.g., $\{111\}$ planes) to construct II-V inward dipole moments that compensate III-VI outward dipole moments at the interfaces (Supplementary Fig. 5). The growth of II-VI shell on these NCs allows to attain III-V/II-VI $h$ NCs with controlled interfacial polarization. The procedures for the synthesis and cation 
exchange, and sample information are detailed in Methods and Supplementary Information (Supplementary Figs. 6 and 7, and Supplementary Table 3 and 4).

The vacuum-level shift by the interfacial polarization is developed in all colloidal IIIV/II-VI $h$-NCs (Fig. 3A). The stoichiometric control by the chemical means permits to engineer the vacuum-levels of InP/ZnSe, InP/ZnS, InAs/ZnSe, In $0.5 \mathrm{Ga} 0.5 \mathrm{P} / \mathrm{ZnSe}$, In $0.3 \mathrm{Ga} 0.7 \mathrm{P} / \mathrm{ZnS}$, and InP/CdS $h$-NCs by 370, 190, 160, 340, 210 and $80 \mathrm{meV}$, respectively (Fig. 3a). We obtained $1 \mathrm{~S}_{\mathrm{hh}}$ values using UPS and $1 \mathrm{~S}_{\mathrm{e}}$ values by taking optical bandgaps obtained from their UV-Vis spectra into account. The measured vacuum-level shift $\left(\Delta E_{V L S}\right)$ in $\mathrm{InP} / \mathrm{ZnSe} h$-NCs is linearly proportional to the interfacial dipole density $(\rho)$ (Fig. 3b).

These results coherently attest that, in addition to the quantum confinement effect, the vacuum-level shift by the interfacial polarization should be taken into account to fully understand the electronic and photophysical properties of III-V/II-VI heterovalent $h$-NCs. This in return offers the possibility to independently customize the electronic characteristics and optical properties of III-V/II-VI $h$-NCs by controlling the interfacial dipoles and their dimensions. For example, the manipulation of the dimension of core materials and the dipole density at the interface allows to modify the electronic energy levels without varying their optical bandgaps (Supplementary Fig. 8). In addition, the facile control awards to alter the optical bandgap and emissive colors without having to change the dimension of $h$-NCs. As an ultimate achievement, by simply varying polarization at the fixed dimension we have obtain a wide range of visible emission colors including the primary colors from $\operatorname{In}_{0.3} \mathrm{Ga}_{0.7} \mathrm{P}(r=1.2 \mathrm{~nm}) / \mathrm{ZnS}(H=2.6 \mathrm{~nm})$, $\operatorname{In} 0.5 \mathrm{Ga} 0.5 \mathrm{P} / \mathrm{ZnSe}(r=1.3 \mathrm{~nm} / H=3.2 \mathrm{~nm})$ and $\mathrm{InP}(r=1.9 \mathrm{~nm}) / \mathrm{ZnSe}(H=2.2 \mathrm{~nm}) h$-NCs (Fig. $3 c)$. 

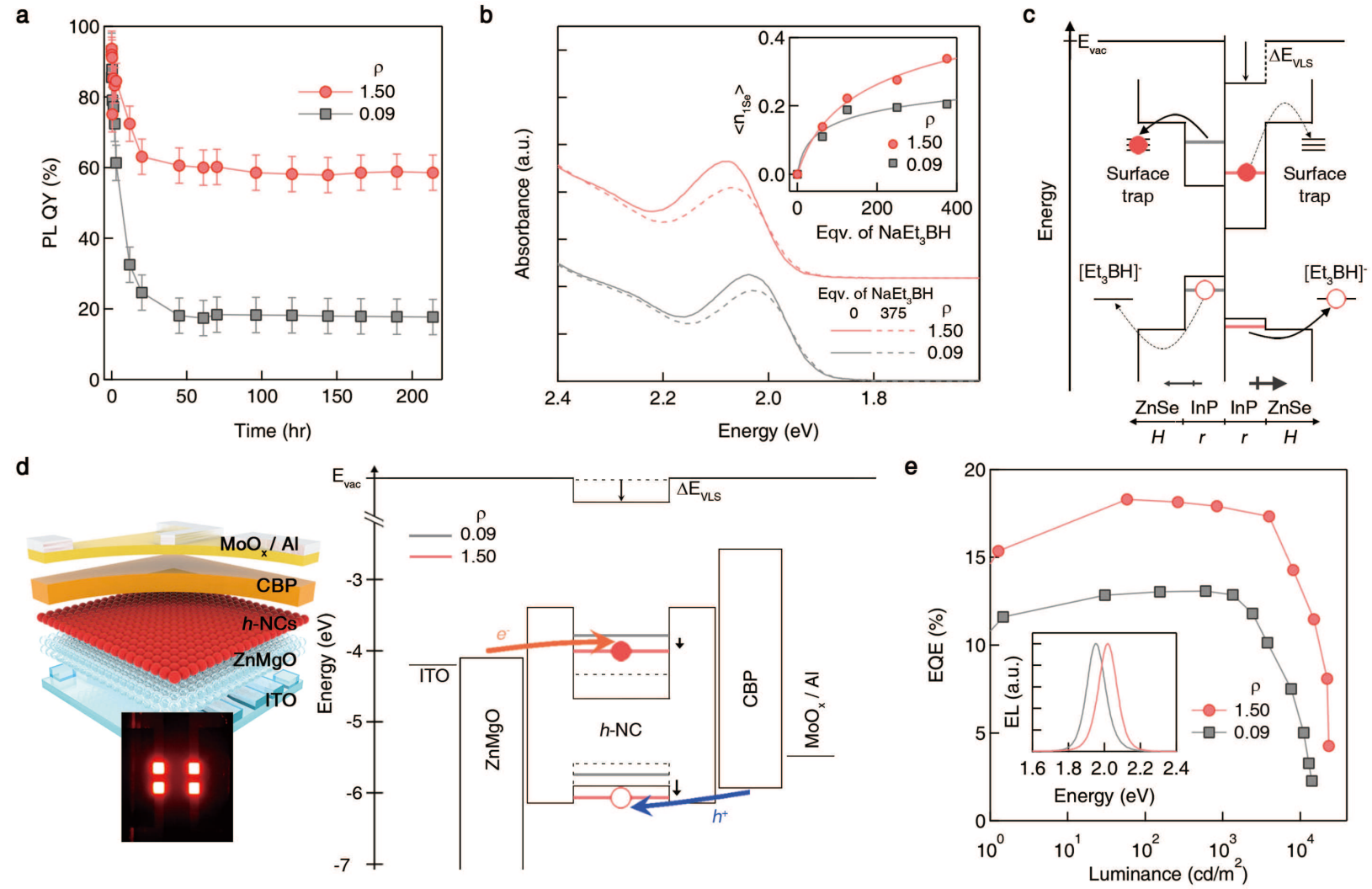

Fig. 4. Impact of the interfacial polarization on transport characteristics of charge carriers across InP/ZnSe heterovalent $\boldsymbol{h}$-NCs. (a) Temporal change in PL QYs of InP/ZnSe $h$-NCs with $\rho=1.50$ (red) versus 0.09 (gray) $\mathrm{nm}^{-2}$ under exposure to air. The average (symbols) and standard deviation (error bars) were calculated from multiple independent runs of the experiment. (b) Absorption spectra of InP/ZnSe $h$-NCs with $\rho=1.50$ (red) versus 0.09 (gray) $\mathrm{nm}^{-2}$ without (solid lines) and with the addition of 375 equivalents of sodium triethylborohydride ( $\left.\mathrm{NaEt}_{3} \mathrm{BH}\right)$ (dotted line). The absorption spectra are vertically shifted for visual clarity. Inset shows the electron occupancy at $1 \mathrm{~S}_{\mathrm{e}}$ as a function of the amount of reductant. (c) Schematic contrasting the injection and ejection characteristics of charge carriers in $h$-NCs with $\rho=1.50$ (left) versus 0.09 (right) $\mathrm{nm}^{-2}$. (d) Schematic illustration and photograph (left) and the energy band diagram of NCLED employing InP/ZnSe $h$-NCs (right). The potential profile and electronic states $\left(1 \mathrm{Se}_{\mathrm{e}}\right.$ and 
1Shh) of InP/ZnSe $h$-NCs with $\rho=1.50$ (red) versus 0.09 (gray) $\mathrm{nm}^{-2}$ are overlayed for comparison. (e) The external quantum efficiency (EQE) versus luminance curves of NC-LEDs employing InP/ZnSe $h$-NCs with $\rho=1.50$ (red) versus 0.09 (gray) $\mathrm{nm}^{-2}$. Inset is the electroluminescence spectra of NC-LEDs employing InP/ZnSe $h$-NCs with $\rho=1.50$ (red) versus 0.09 (gray) $\mathrm{nm}^{-2}$.

The potential profile and the energy level offset of $h$-NCs govern the injection and emission properties of charge carriers that determine the photochemical characteristics of $h$-NCs. Specifically, electrons trapped at the surface states are responsible for the irreversible efficiency loss of InP/ZnSe $h$-NCs under exposure to air for an extended period ${ }^{25}$. The presence of outward dipole moments at the InP/ZnSe interface lowers the electronic energy levels and raises the energy barrier for electron emission to the surface trap states. The change in the potential profile impedes the photo-generated electrons to be trapped at the surface trap states and enhances the oxidative stability of $h$-NCs accordingly (Fig. 4a). Similarly to the oxidative test, the redox properties of InP/ZnSe $h$-NCs depend upon the interface stoichiometry. The first excitonic absorption band of $\mathrm{InP} / \mathrm{ZnSe} h$-NCs bleaches with the addition of reductants (i.e., sodium triethylborohydride, $\mathrm{NaEt}_{3} \mathrm{BH}$ ) under UV illumination, which indicates the presence of excess electrons in the $1 \mathrm{~S}_{\mathrm{e}}$ state of $h$-NCs by scavenging a hole from a photo-generated electron-hole pair $^{34,35}$. InP/ZnSe $h$-NCs with greater outward dipole densities retain less potential barrier for hole extraction and thus exhibit accelerated bleaching rates upon the addition of reductant (Fig. 4b, Supplementary Fig. 9).

Furthermore, the transport characteristics of charge carriers across the heterostructure have a significant bearing on the performance of electronic devices employing $h$-NCs. Here, we 
examine the performance of NC based light-emitting diodes (NC-LEDs), in which InP/ZnSe $h$ NCs convert charge carriers into photons (Fig. 4d, e). NC-LEDs are constructed in an inverted structure of ITO // ZnMgO // InP/ZnSe $h$-NCs with varying interfacial dipole densities ( $\rho=1.50$ and $0.09 \mathrm{~nm}^{-2}$ ) // 4,4'-bis(N-carbazolyl)-1,1'-biphenyl (CBP) // $\mathrm{MoO}_{\mathrm{x}} / / \mathrm{Al}$ (Fig. 4c). $\mathrm{ZnMgO}$ and CBP serve as an electron transport layer (ETL) and a hole transport layer (HTL), respectively, and $\mathrm{MoO}_{\mathrm{x}}$ assists the efficient injection of holes from $\mathrm{Al}$ (cathode) into CBP. In such a device configuration, the hole injection from HTL into InP/ZnSe $h$-NCs takes place rather spontaneously, whereas the electron injection from ETL into InP/ZnSe $h$-NCs requires the assistance of external electric field ${ }^{24,26}$. The inequality in the charge injection rates often yields the presence of excess holes in $h$-NCs that reduces the luminescence efficiency of the NC emissive layer within the working devices by promoting non-radiative Auger recombination $\operatorname{processes}^{24,26}$.

The down-shift of energy levels with the insertion of outward interfacial polarization reduces the energy offset between the conduction band edge of $\mathrm{ZnMgO}$ and $1 \mathrm{~S}_{\mathrm{e}}$ of $\mathrm{InP} / \mathrm{ZnSe} h$ $\mathrm{NCs}\left(\Delta\right.$ Eelectron $=660$ and $260 \mathrm{meV}$ for $\mathrm{InP} / \mathrm{ZnSe} h$-NCs with $\rho=0.09$ and $1.50 \mathrm{~nm}^{-2}$, respectively), and thus expedites the electron injection from $\mathrm{ZnMgO}$ into $h$-NCs (Supplementary Fig. 10). Likewise, the outward dipole enlarges the energy offset between the valence band edge of CBP and $1 \mathrm{~S}$ hh of InP/ZnSe $h$-NCs $\left(\Delta \mathrm{E}_{\text {hole }}=-160\right.$ and $190 \mathrm{meV}$ for $\mathrm{InP} / \mathrm{ZnSe} h$-NCs with $\rho=$ 0.09 and $1.50 \mathrm{~nm}^{-2}$, respectively) and impedes the hole injection from CBP into $h$-NCs (Supplementary Fig. 10). These changes in the energy positions of InP/ZnSe $h$-NCs restore the balance of the electron and hole injection rate into the $h$-NC emissive layer and consequently enhance the luminance efficiency of NC-LEDs (Fig. 4e, Supplementary Table 5). Specifically, the peak external quantum efficiency of NC-LEDs boosts from $13.07 \%$ to $18.30 \%$ as the 
interfacial dipole density increases from $0.09 \mathrm{~nm}^{-2}$ to $1.50 \mathrm{~nm}^{-2}$. Considering no significant difference in PL QY observed among the series of $\mathrm{InP} / \mathrm{ZnSe} h$-NCs, the enhancement in the device efficiency arises from the balanced electron and hole injection rate into InP/ZnSe $h$-NCs within the device. The reduction of driving voltage as well as the rise of peak brightness of NCLEDs are also the outcomes of the engineered heterovalent interface (Supplementary Table 5). It is noteworthy that, given the outcoupling efficiency of $20 \%$, the obtained device efficiency is close to the theoretical limit.

We observed the interfacial polarization induced by heterovalent bondings at the interface of III-V/II-VI $h$-NCs. The potential changes at $h$-NCs by the interfacial polarization appears to far exceed those created in bulk semiconductors. Obvious implication is that the control of heterovalent bondings relates profoundly to the optical and electrical properties of $h$ NCs. In addition, we have offered an effective chemical means to control the interfacial polarization and exemplified how to boost photochemical stability and optoelectronic performance of $h$-NCs. Our results suggest that, in chorus with the quantum confinement effect, the capability of engineering the structural characteristics of the heterovalent interfaces allows one to customize the potential profile of heavy metal-free III-V/II-VI $h$-NCs that overcome the limitation of materials choice for LEDs. Therefore, our work showing the findings and advances in materials engineering will be applied to the practicable use of III-V/II-VI $h$-NCs in a wide range of light-emitting devices. 


\section{Methods:}

Materials. Indium acetate $\left(\operatorname{In}(\mathrm{ac})_{3}, 99.99 \%\right)$, zinc acetate $\left(\mathrm{Zn}(\mathrm{ac})_{2}, 99.99 \%\right)$, oleic acid (OA, 99\%), 1-octadecene (ODE, 99\%), tris(trimethylsilyl)phosphine ((TMS) 3 P, 99.9\%), ntrioctylphosphine (TOP, 99\%), selenium (Se, 99.9\%), sulfur (S, 99.9\%) were purchased from Uniam. Tris(trimethylsilyl)arsine ((TMS) $\left.{ }_{3} \mathrm{As}, 99 \%\right)$ was purchased from JSI Silicone and distilled before use. Zinc chloride $\left(\mathrm{ZnCl}_{2}, 99.999 \%\right.$, trace metal basis), cadmium oxide (CdO, 99.5\%, trace metals basis), cadmium acetate $(\mathrm{Cd}(\mathrm{ac}) 2,99.995 \%)$, cadmium chloride $\left(\mathrm{CdCl}_{2}\right.$, 99.99\%, trace metals basis), dioctylamine (DOA, 97\%) and 1-dodecanethiol (DDT, $\geq 98 \%$ ) were purchased from Sigma-Aldrich. $\mathrm{GaCl}_{3}(99.999 \%$, metals basis) was purchased from Alfa aesar. All organic solvents were purchased from Daejung. All chemicals, unless otherwise stated, were used as received.

Preparation of precursor solutions. All chemicals were treated under an inert atmosphere. 0.5 $\mathrm{M}$ indium oleate $\left(\operatorname{In}(\mathrm{OA})_{3}\right)$, zinc oleate $\left(\mathrm{Zn}(\mathrm{OA})_{2}\right)$ and cadmium oleate $\left(\mathrm{Cd}(\mathrm{OA})_{2}\right)$ stock solutions in ODE were prepared for cation precursors and 2M TOPSe and TOPS were prepared for anion precursors. For the preparation of $\operatorname{In}(\mathrm{OA})_{3}, 50 \mathrm{mmol}$ of $\operatorname{In}(\mathrm{ac})_{3}$ and $150 \mathrm{mmol}$ of OA were mixed in a 3-neck flask and degassed at $130{ }^{\circ} \mathrm{C}$ for 3 hours. The precursor was then diluted to $0.5 \mathrm{M}$ concentration with $\mathrm{ODE}$. $\mathrm{Zn}(\mathrm{OA})_{2}$ was made through a similar procedure. For $\mathrm{Cd}(\mathrm{OA})_{2}$ stock solution, $50 \mathrm{mmol}$ of $\mathrm{CdO}, 150 \mathrm{mmol}$ of OA were placed in a 3-neck flask and degassed at $110^{\circ} \mathrm{C}$ for 1 hour. After the backfilling of nitrogen, the flask was heated up to $240{ }^{\circ} \mathrm{C}$ for 3 hours. The precursor was diluted with ODE to $0.5 \mathrm{M}$ concentration. TOPSe and TOPS were prepared in a nitrogen-filled glove box. $200 \mathrm{mmol}$ of Se (or S) was mixed with $100 \mathrm{~mL}$ of TOP 
and heated up to $180{ }^{\circ} \mathrm{C}$ for 2 hours. After the reaction solution becomes transparent, the precursors cooled down to room temperature. All precursors are stored under an inert condition prior to use.

Synthesis of InP NCs. Preparation of InP cores proceeded on the previously reported method with minor modifications ${ }^{25}$. A mixture of $2 \mathrm{ml}$ of $0.5 \mathrm{M} \operatorname{In}(\mathrm{OA})_{3}$ stock solution and $29 \mathrm{ml}$ of ODE were loaded in a 3-neck flask and degassed at $110{ }^{\circ} \mathrm{C}$ for 1 hour under vacuum. The flask was filled with nitrogen before the injection of phosphorus precursors. A phosphorus solution (a mixture of $0.5 \mathrm{mmol}$ of (TMS) $)_{3} \mathrm{P}$ diluted with $1 \mathrm{~mL}$ of TOP) was injected into the reaction flask to form In-P complexes. After the injection of phosphorus precursor, the flask was heated up to $260{ }^{\circ} \mathrm{C}$ to form InP nuclei and kept the reaction temperature for $10 \mathrm{~min}$. The smallest $(r=1.3$ $\mathrm{nm}$ ) InP cores can be obtained. For further growth of InP core, the proper amount of In and P precursors were injected dropwise at the same temperature. The crude solution was purified via the precipitation/redispersion method with toluene and acetone. The resulting NCs were dissolved in toluene at a concentration of $100 \mathrm{mg} / \mathrm{mL}$ for further characterization and shell growth.

Synthesis of InGaP NCs. $2 \mathrm{~mL}$ of $0.5 \mathrm{M} \operatorname{In}(\mathrm{OA})_{3}$ stock solution, $2 \mathrm{mmol}$ (or desired amount) of $\mathrm{GaCl}_{3}, 6 \mathrm{mmol}$ of $\mathrm{OA}$, and $20 \mathrm{~mL}$ of ODE were placed in a 3-neck flask and degassed at $110^{\circ} \mathrm{C}$ for 1 hour. After filling nitrogen, the reaction flask was heated up to $180^{\circ} \mathrm{C}$. The phosphorous solution was rapidly injected into the flask and kept for 10 min under vigorous mixing. The temperature of the reaction flask was raised up to $260^{\circ} \mathrm{C}$ and maintained for $30 \mathrm{~min}$. The flask was cooled down to quench the reaction. The resulting NCs were purified twice via precipitation 
(acetone)/redispersion (toluene) method and dispersed in toluene at a concentration of 100 $\mathrm{mg} / \mathrm{mL}$ for further characterization and shell growth.

Synthesis of InAs NCs. InAs NCs were prepared via the continuous injection method with minor modifications ${ }^{36}$. First, we prepared amorphous InAs nanoclusters. $0.84 \mathrm{~g}$ of $\left(\mathrm{TMS}_{3}\right) \mathrm{As}$, $2.19 \mathrm{~g}$ of DOA and $6 \mathrm{~mL}$ of ODE were mixed at $60{ }^{\circ} \mathrm{C}$ in a glove box and kept the temperature for 1 hour to form arsenic stock solution. $12 \mathrm{~mL}$ of $0.5 \mathrm{M} \mathrm{In}(\mathrm{OA})_{3}$ and $30 \mathrm{~mL}$ of ODE were degassed at $110^{\circ} \mathrm{C}$ for 2 hours in a 3-neck flask. After backfilling with nitrogen, the temperature of the flask lowered to the room temperature. The arsenic solution was injected into the reaction flask and kept for $10 \mathrm{~min}$. For the preparation of InAs seeds, $4 \mathrm{~mL}$ of $0.5 \mathrm{M} \operatorname{In}(\mathrm{OA})_{3}$ and $10 \mathrm{~mL}$ of ODE were degassed at $110^{\circ} \mathrm{C}$ for 2 hours. The reaction flask was filled with nitrogen and the temperature was raised up to $300{ }^{\circ} \mathrm{C}$. The arsenic stock solution was injected into the flask to form InAs seeds. The reaction continued for 20 mins at the elevated temperature. For further growth of the InAs NCs, the desired amount of InAs nanocluster solution was injected into the InAs seeds solution dropwise at a rate of $0.05 \mathrm{mmol} / \mathrm{min}$. After the growth of InAs NCs, the flask was cooled down to the room temperature. The resulting NCs were purified twice via precipitation (butanol)/redispersion (hexane) method and dispersed in toluene at a concentration of $100 \mathrm{mg} / \mathrm{mL}$ for further characterization and shell growth.

Cation exchange process. The In:P stoichiometry of InP cores in InP/ZnSe $h$-NCs was controlled via the cation exchange process prior to the growth of ZnSe shell. For the typical cation exchange process from In to $\mathrm{Zn}$ on the surface of $\mathrm{InP} N C s$, the desired amount of $\mathrm{Zn}(\mathrm{Cl})_{2}$ (or $\mathrm{Zn}(\mathrm{ac})_{2}$ ) and $2 \mathrm{~mL}$ of $0.5 \mathrm{M} \mathrm{Zn}(\mathrm{OA})_{2}$ in $10 \mathrm{~mL}$ of ODE were placed in a 3-neck flask and 
degassed under vacuum. After the backfilling of nitrogen, the reaction flask heated up to $180{ }^{\circ} \mathrm{C}$ before $0.5 \mathrm{~mL}$ of InP core solution $(r=1.9 \mathrm{~nm}$, concentration $=100 \mathrm{mg} / \mathrm{ml})$ was injected. The reaction temperature was kept for $30 \mathrm{~min}$ to complete the cation exchange. The stoichiometry of In:P can be precisely tuned by changing the amount of reactive $\mathrm{Zn}$ precursors $\left(\mathrm{Zn}(\mathrm{Cl})_{2}\right.$ or $\left.\mathrm{Zn}(\mathrm{ac})_{2}\right)$. For example, the In:P ratio of the pristine InP NCs was 1.12:1.00, and the cation exchange with 1 and $3 \mathrm{mmol}$ of reactive $\mathrm{Zn}$ precursors yielded In:P ratio of 1.09:1.00, and 1.07:1.00, respectively. Similarly, incorporating reactive $\mathrm{Cd}$ precursors $\left(\mathrm{Cd}(\mathrm{Cl})_{2}\right.$ or $\left.\mathrm{Cd}(\mathrm{ac})_{2}\right)$ promotes the cation exchange process from In to $\mathrm{Cd}$. The interfacial stoichiometry in InAs/ZnSe, InGaP/ZnSe, and InGaP/ZnS $h$-NCs also can be controlled via the analogous cation exchange processes.

Synthesis of core/shell heterostructured nanocrystals (h-NCs). Core/shell heterovalent $h$-NCs were synthesized via the previously reported method with modifications ${ }^{25}$. For the synthesis of $\mathrm{InP} / \mathrm{ZnSe} h$-NCs, $2 \mathrm{~mL}$ of $\mathrm{Zn}(\mathrm{OA})_{2}$ stock solution, and $10 \mathrm{~mL}$ of ODE were loaded in a 3-neck flask. The flask was degassed at $110^{\circ} \mathrm{C}$ for 2 hours. After filling up with nitrogen, the flask was heated up to $180^{\circ} \mathrm{C} .0 .5 \mathrm{~mL}$ of $\mathrm{InP}$ cores $(100 \mathrm{mg} / \mathrm{ml})$ was injected into the flask. The reaction temperature was kept at $180{ }^{\circ} \mathrm{C}$ for $30 \mathrm{~min}$ to evaporate the solvent. The reaction flask was heated up to $300{ }^{\circ} \mathrm{C}$ and $0.4 \mathrm{~mL}$ of TOPSe was added dropwise to grow ZnSe shell. After 1 hour of the reaction, the flask was cooled down to the room temperature. The resulting $h$-NCs were purified twice via the precipitation (ethanol)/redispersion (toluene) method. $h$-NCs were dispersed in toluene at a concentration of $100 \mathrm{mg} / \mathrm{mL}$ for further characterizations. The use of TOPS instead of TOPSe yielded InP/ZnS $h$-NCs. For the growth of CdS shell on the InP NCs, $\mathrm{Cd}(\mathrm{OA})_{2}$ stock solution and DDT were used instead of $\mathrm{Zn}(\mathrm{OA})_{2}$ and TOPSe, respectively. 
$\mathrm{InP} / \mathrm{ZnS}, \operatorname{InAs} / \mathrm{ZnSe}, \mathrm{In}_{0.5} \mathrm{Ga} 0.5 \mathrm{P} / \mathrm{ZnSe}, \mathrm{In}_{0.3} \mathrm{Ga}{ }_{0.7} \mathrm{P} / \mathrm{ZnS}$, and $\mathrm{InP} / \mathrm{CdS} h$-NCs were synthesized via the analogous procedure.

Characterization. UV-Vis absorption and visible photoluminescence (PL) spectra were collected with UV-1800 (Shimadzu), and a Fluoromax-4 spectrometer (Horiba Science), respectively. Vis-NIR absorption and NIR PL spectra were obtained with UV-3600 Plus (Shimadzu) and an FLS 1000 photoluminescence spectrometer (Edinburgh Instruments Ltd). The photoluminescence quantum yield (PL QY) of NCs (dispersed in toluene) was measured with the QE-2000 (Otsuka Electronics). For the absolute PL QY measurement of $h$-NC in solution, we set the optical density of NCs to 0.1 at excitation wavelength $450 \mathrm{~nm}$. PL decay dynamics were obtained with a time-correlated single-photon counting (TCSPC) system (Picoquant) with the resolution of $\sim 100 \mathrm{ps}$. The samples were excited at $2.76 \mathrm{eV}$ at a repetition rate of $0.5 \mathrm{MHz}$. We obtained TEM images from LIBRA 120 (tungsten filament, ZEISS) and Tecnai-F20 (FEI). The radius of InP core was estimated from the empirical equation of band gap energy versus the diameter of InP NCs ${ }^{37}$. The size of $h$-NCs was measured from TEM images (averaged values over hundreds of $h$-NCs). The chemical composition of InP, InP/ZnSe and other $h$-NCs was obtained by the elemental analysis based on inductively coupled plasma atomic emission spectrometer (ICP-AES, VARIAN ICP-730ES). The ultraviolet and x-ray photoemission spectra of III-V/II-VI $h$-NCs were collected with NEXSA (ThermoFisher Scientific), using illumination of He (I) irradiation $(h v=21.22 \mathrm{eV})$ for UPS and Al Ka radiation $(h v=1486.6 \mathrm{eV})$ for XPS. The binding energy was calibrated using the $\mathrm{C} 1 \mathrm{~s}$ peak at $284.8 \mathrm{eV}$ from C-C bonds of surface ligands. $h$-NC solutions ( $23 \mathrm{mg} / \mathrm{ml}$ in octane) were spun-cast onto the thermally evaporated $\mathrm{Cr}$ 
$(10 \mathrm{~nm}) / \mathrm{Au}(50 \mathrm{~nm}) / \mathrm{Si}(100)$ substrates to achieve $h$-NC films for UPS and XPS measurement. The samples were dried under vacuum for 30 min prior to the measurement.

Fabrication of nanocrystal-light emitting devices (NC-LEDs). Patterned indium tin oxide (ITO) on glass was used as the substrate of NC-LEDs. The substrate was cleaned with acetone, isopropyl alcohol, and deionized water, sequentially. The substrates were dried in $90{ }^{\circ} \mathrm{C}$ drying oven for $30 \mathrm{~min}$ prior to deposition of other layers. $20 \mathrm{mg} / \mathrm{mL}$ of $\mathrm{ZnMgO}$ NP solution dispersed in ethanol was spun-cast on the substrate at a rate of 2,000 rpm for $30 \mathrm{sec}$ to from the $\mathrm{ZnMgO}$ electron transport layer. $h$-NC solutions $(13 \mathrm{mg} / \mathrm{mL}$ in n-octane) were spun-cast at 4,000 rpm for $30 \mathrm{sec}$ to form the $\mathrm{NC}$ emissive layer. $\mathrm{CBP}, \mathrm{MoO}_{\mathrm{x}}$, and $\mathrm{Al}$ layer was thermally evaporated on the $\mathrm{NCs} / / \mathrm{ZnMgO} / / \mathrm{ITO}$ substrates under a vapor pressure of $5 \times 10^{-7}$ Torr at deposition rates of 1 , 0.1-0.2, and 3-4 $\AA \cdot \mathrm{s}^{-1}$, respectively. For electron-only device, Al layer was thermally evaporated on the $\mathrm{ZnMgO} / / \mathrm{NCs} / / \mathrm{ZnMgO} / / \mathrm{ITO}$ substrates at a deposition rate of $3-4 \AA \cdot \mathrm{s}^{-1}$. For hole only device, PEDOT:PSS solution was spun cast on the ITO substrates at 5,000 rpm for $30 \mathrm{sec}$, followed by the deposition of $\mathrm{NC}$ emissive layers. $\mathrm{CBP}, \mathrm{MoO}_{\mathrm{x}}$ and $\mathrm{Al}$ layer was thermally evaporated on the NCs//PEDOT:PSS//ITO under a vapor pressure of $5 \times 10^{-7}$ Torr at deposition rates of $1,0.1-0.2$, and $3-4 \AA \cdot \mathrm{s}^{-1}$, respectively. The current-voltage-luminance $(J-V-L)$ characteristics of NC-LEDs were measured using a spectroradiometer (Konica Minolta CS-2000) coupled with a source meter unit (SMU 238, Keithley instruments).

Data availability: The authors declare that all data supporting this work are contained in graphics displayed in the main text or in supplementary information. Correspondence and requests for materials should be addressed to corresponding authors. 
Acknowledgments: We thank S. M. Park and S. Jeong for synthesis and characterization of InAs NCs. This work was supported by the National Research Foundation of Korea (NRF) grant funded by the Ministry of Science, ICT, and Future Planning (NRF-2020R1A2C2011478, NRF2020M3D1A2101310, NRF-2020M3H4A1A01086888), the Technology Innovation Program (20010737) funded by the Ministry of Trade, Industry \& Energy (MOTIE, Korea), and the grant funded by Samsung Display.

\section{Author information:}

$†$ These authors contributed equally to this work: Byeong Guk Jeong, Jun Hyuk Chang, Donghyo Hahm

Affiliations:

SKKU Advanced Institute of Nanotechnology and Department of Nano Engineering, Sungkyunkwan University, Suwon 16419, Republic of Korea

Byeong Guk Jeong, Jun Hyuk Chang, Donghyo Hahm, Seunghyun Rhee, Youngdu Kim, Doyoon Shin, Jeong Woo Park, Euyheon Hwang, and Wan Ki Bae

Samsung Display Research Center, Samsung Display, Yongin 17113, Republic of Korea Myeongjin Park, Sooho Lee, and Changhee Lee

Department of Chemical and Biomolecular Engineering, KAIST Institute for the Nanocentury, Korea Advanced Institute of Science and Technology (KAIST), Daejeon 34141, Republic of Korea

Doh C. Lee 
Display Research Center, Korea Electronics Technology Institute (KETI), Seongnam 13509, Republic of Korea.

Kyoungwon Park

\section{Contributions}

B.G.J., J.H.C., D.H., S.H.R, D.C.L., K.P., E.H., and W.K.B. conceived the original idea and designed the experiments; B.G.J., J.H.C., D.H., D.S., J.W.P. conducted synthesis and characterization of NCs and analyzed the experimental data; J.H.C., Y.K., K.P., and E.H. carried out the computational calculation. S.H.R., S.L., M.P., and C.L. fabricated all devices and analyzed the data. All authors contributed in the preparation of the manuscript.

\section{Corresponding Authors}

Correspondence to Wan Ki Bae (wkbae@skku.edu); Euyheon Hwang (euyheon@skku.edu);

Doh C. Lee (1clee@,kaist.edu $)$

Competing interests: The authors declare that they have no competing interests.

\section{Additional Information}

Publisher's note Springer Nature remains neutral with regard to jurisdictional claims in published maps and institutional affiliations.

\section{Supplementary information}

Supplementary Notes 1 and 2, Supplementary Figs. 1-10 and Supplementary Tables 1-5. 


\section{References}

1. Brus, L. Electronic wave functions in semiconductor clusters: experiment and theory. J. Phys. Chem. 90, 2555-2560 (1986).

2. Ekimov, A. I., Efros, A. L. \& Onushchenko, A. A. Quantum size effect in semiconductor microcrystals. Solid State Commun. 56, 921-924 (1985).

3. Murray, C. B., Norris, D. J. \& Bawendi, M. G. Synthesis and characterization of nearly monodisperse $\mathrm{CdE}(\mathrm{E}=$ sulfur, selenium, tellurium) semiconductor nanocrystallites. J. Am. Chem. Soc. 115, 8706-8715 (1993).

4. Pietryga, J. M. et al. Spectroscopic and device aspects of nanocrystal quantum dots. Chem. Rev. 116, 10513-10622 (2016).

5. Park, Y.-S., Lim, J. \& Klimov, V. I. Asymmetrically strained quantum dots with nonfluctuating single-dot emission spectra and subthermal room-temperature linewidths. Nat. Mater. 18, 249-255 (2019).

6. Colvin, V. L., Schlamp, M. C. \& Alivisatos, A. P. Light-emitting diodes made from cadmium selenide nanocrystals and a semiconducting polymer. Nature 370, 354-357 (1994).

7. Wang, Y. \& Herron, N. Photoconductivity of CdS nanocluster-doped polymers. Chem. Phys. Lett. 200, 71-75 (1992).

8. McDonald, S. A. et al. Solution-processed $\mathrm{PbS}$ quantum dot infrared photodetectors and photovoltaics. Nat. Mater. 4, 138-142 (2005).

9. Klimov, V. I. et al. Optical gain and stimulated emission in nanocrystal quantum dots. Science 290, 314 (2000).

10. Lim, J., Park, Y.-S. \& Klimov, V. I. Optical gain in colloidal quantum dots achieved with direct-current electrical pumping. Nat. Mater. 17, 42-49 (2018). 
11. Meinardi, F. et al. Large-area luminescent solar concentrators based on 'Stokes-shiftengineered' nanocrystals in a mass-polymerized PMMA matrix. Nat. Photonics 8, 392-399 (2014).

12. Meinardi, F. et al. Highly efficient large-area colourless luminescent solar concentrators using heavy-metal-free colloidal quantum dots. Nat. Nanotechnol. 10, 878-885 (2015).

13. Bruchez, M., Moronne, M., Gin, P., Weiss, S. \& Alivisatos, A. P. Semiconductor nanocrystals as fluorescent biological labels. Science 281, 2013-2016 (1998).

14. Chan, W. C. W. \& Nie, S. Quantum dot bioconjugates for ultrasensitive nonisotopic detection. Science 281, 2016 (1998).

15. Michalet, X. et al. Quantum dots for live cells, in vivo imaging, and diagnostics. Science 307, 538-544 (2005).

16. Medintz, I. L., Uyeda, H. T., Goldman, E. R. \& Mattoussi, H. Quantum dot bioconjugates for imaging, labelling and sensing. Nat. Mater. 4, 435-446 (2005).

17. Hines, M. A. \& Guyot-Sionnest, P. Synthesis and characterization of strongly luminescing ZnS-capped CdSe nanocrystals. J. Phys. Chem. 100, 468-471 (1996).

18. Kim, S., Fisher, B., Eisler, H.-J. \& Bawendi, M. Type-II quantum dots:

$\mathrm{CdTe} / \mathrm{CdSe}$ (core/shell) and CdSe/ZnTe(core/shell) heterostructures. J. Am. Chem. Soc. 125, 11466-11467 (2003).

19. García-Santamaría, F. et al. Suppressed Auger recombination in "Giant" nanocrystals boosts optical gain performance. Nano Lett. 9, 3482-3488 (2009).

20. Haubold, S., Haase, M., Kornowski, A. \& Weller, H. Strongly luminescent InP/ZnS coreshell nanoparticles. ChemPhysChem 2, 331-334 (2001). 
21. Bae, W. K. et al. Controlling the influence of Auger recombination on the performance of quantum-dot light-emitting diodes. Nat. Commun. 4, 2661 (2013).

22. Yang, Y. et al. High-efficiency light-emitting devices based on quantum dots with tailored nanostructures. Nat. Photonics 9, 259-266 (2015).

23. Cao, W. et al. Highly stable QLEDs with improved hole injection via quantum dot structure tailoring. Nat. Commun. 9, 2608 (2018).

24. Lim, J. et al. Highly efficient cadmium-free quantum dot light-emitting diodes enabled by the direct formation of excitons within InP@ZnSeS quantum dots. ACS Nano 7, 9019-9026 (2013). 25. Hahm, D. et al. Design principle for bright, robust, and color-pure $\mathrm{InP} / \mathrm{ZnSexS} 1-\mathrm{x} / \mathrm{ZnS}$ heterostructures. Chem. Mater. 31, 3476-3484 (2019).

26. Won, Y.-H. et al. Highly efficient and stable $\mathrm{InP} / \mathrm{ZnSe} / \mathrm{ZnS}$ quantum dot light-emitting diodes. Nature 575, 634-638 (2019).

27. Hahm, D. et al. Environmentally benign nanocrystals: challenges and future directions. J. Inf. Disp. 20, 61-72 (2019).

28. Reiss, P., Carrière, M., Lincheneau, C., Vaure, L. \& Tamang, S. Synthesis of semiconductor nanocrystals, focusing on nontoxic and earth-abundant materials. Chem. Rev. 116, 10731-10819 (2016).

29. Kley, A. \& Neugebauer, J. Atomic and electronic structure of the GaAs/ZnSe(001) interface. Phys. Rev. B 50, 8616-8628 (1994).

30. Tung, R. T. \& Kronik, L. Charge density and band offsets at heterovalent semiconductor interfaces. Adv. Theory Simul. 1, 1700001 (2018). 
31. Zehner, R. W., Parsons, B. F., Hsung, R. P. \& Sita, L. R. Tuning the work function of gold with self-assembled monolayers derived from $\mathrm{X}-[\mathrm{C} 6 \mathrm{H} 4-\mathrm{C}: \mathrm{C}-] \mathrm{nC} 6 \mathrm{H} 4-\mathrm{SH}(\mathrm{n}=0,1,2 ; \mathrm{X}=\mathrm{H}$, F, CH3, CF3, and OCH3). Langmuir 15, 1121-1127 (1999).

32. Kirkwood, N. et al. Finding and fixing traps in II-VI and III-V colloidal quantum dots: the importance of Z-type ligand passivation. J. Am. Chem. Soc. 140, 15712-15723 (2018).

33. Friedfeld, M. R. et al. Effects of $\mathrm{Zn} 2+$ and Ga3+ doping on the quantum yield of clusterderived InP quantum dots. J. Chem. Phys. 151, 194702 (2019).

34. Rinehart, J. D., Schimpf, A. M., Weaver, A. L., Cohn, A. W. \& Gamelin, D. R. Photochemical electronic doping of colloidal CdSe nanocrystals. J. Am. Chem. Soc. 135, 1878218785 (2013).

35. Carroll, G. M., Tsui, E. Y., Brozek, C. K. \& Gamelin, D. R. Spectroelectrochemical measurement of surface electrostatic contributions to colloidal CdSe nanocrystal redox potentials. Chem. Mater. 28, $7912-7918$ (2016).

36. Song, J. H., Choi, H., Pham, H. T. \& Jeong, S. Energy level tuned indium arsenide colloidal quantum dot films for efficient photovoltaics. Nat. Commun. 9, 4267 (2018).

37. Cho, E., Jang, H., Lee, J. \& Jang, E. Modeling on the size dependent properties of InP quantum dots: a hybrid functional study. Nanotechnology 24, 215201 (2013). 


\section{Figures}

a
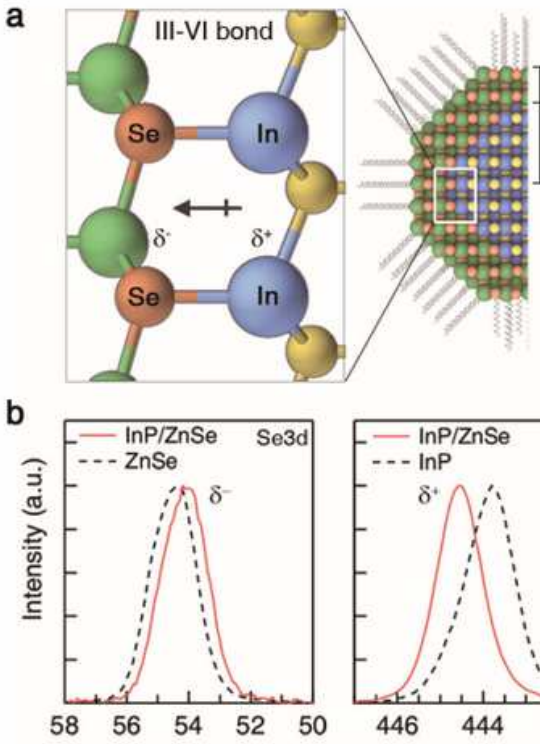

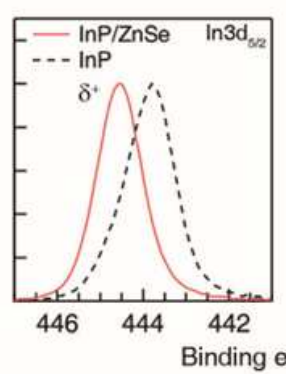

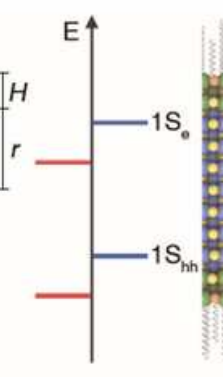

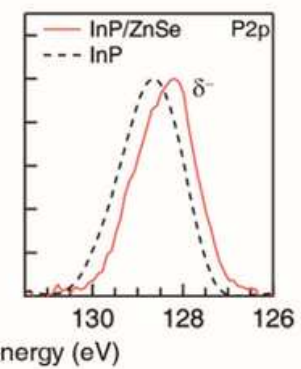

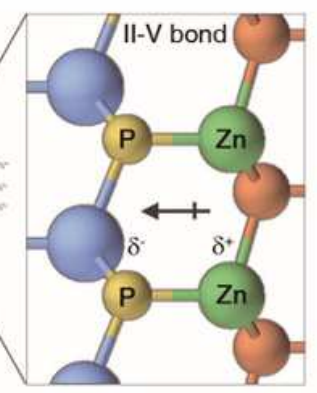

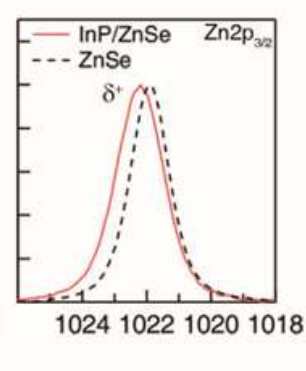

C

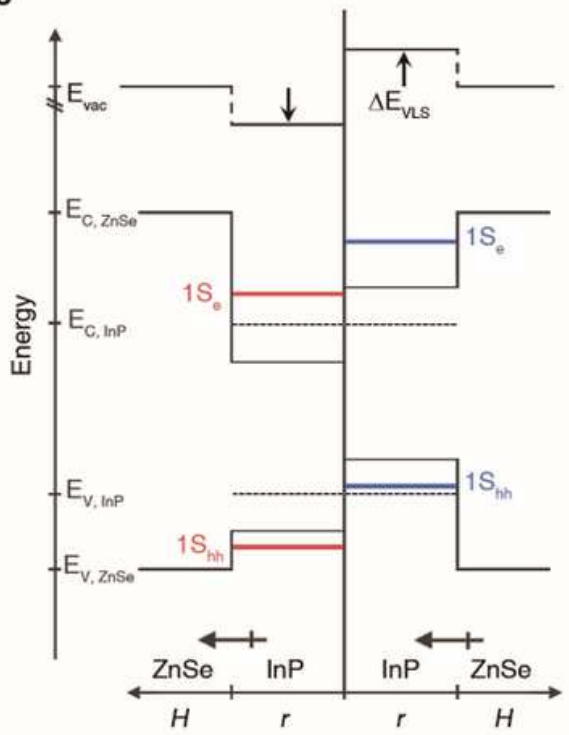

Figure 1

Formation of bond dipoles at the InP/ZnSe interface and their impact on the potential profile of h-NCs. (a) Schematics illustrating bond dipoles at the (111) interface of zinc blende InP/ZnSe h-NCs. The In-Se (left) and Zn-P (right) bonds create outward and inward dipole moments, respectively. The interfacial dipole moments accompany the change in the electronic energy levels of h-NCs. (b) X-ray photoemission spectra (XPS) of Se3d, In3d5/2, P2p, and Zn2p3/2 obtained from InP/ZnSe h-NCs (red solid lines). XPS spectra of In3d5/2 and P2p obtained from InP NCs and Se3d and Zn2p3/2 obtained from ZnSe NCs are overlayed for comparison (black dashed lines). (c) Schematic illustration of the change in the potential profile of InP/ZnSe h-NCs in the presence of outward (left) versus inward (right) dipole moments at the heterovalent interface. The dipole moments at the interface cause the vacuum-level shift that alters the electronic energy levels. The lowest quantized states of conduction band (1Se) and valance band (1Shh) are depicted with colored lines. 

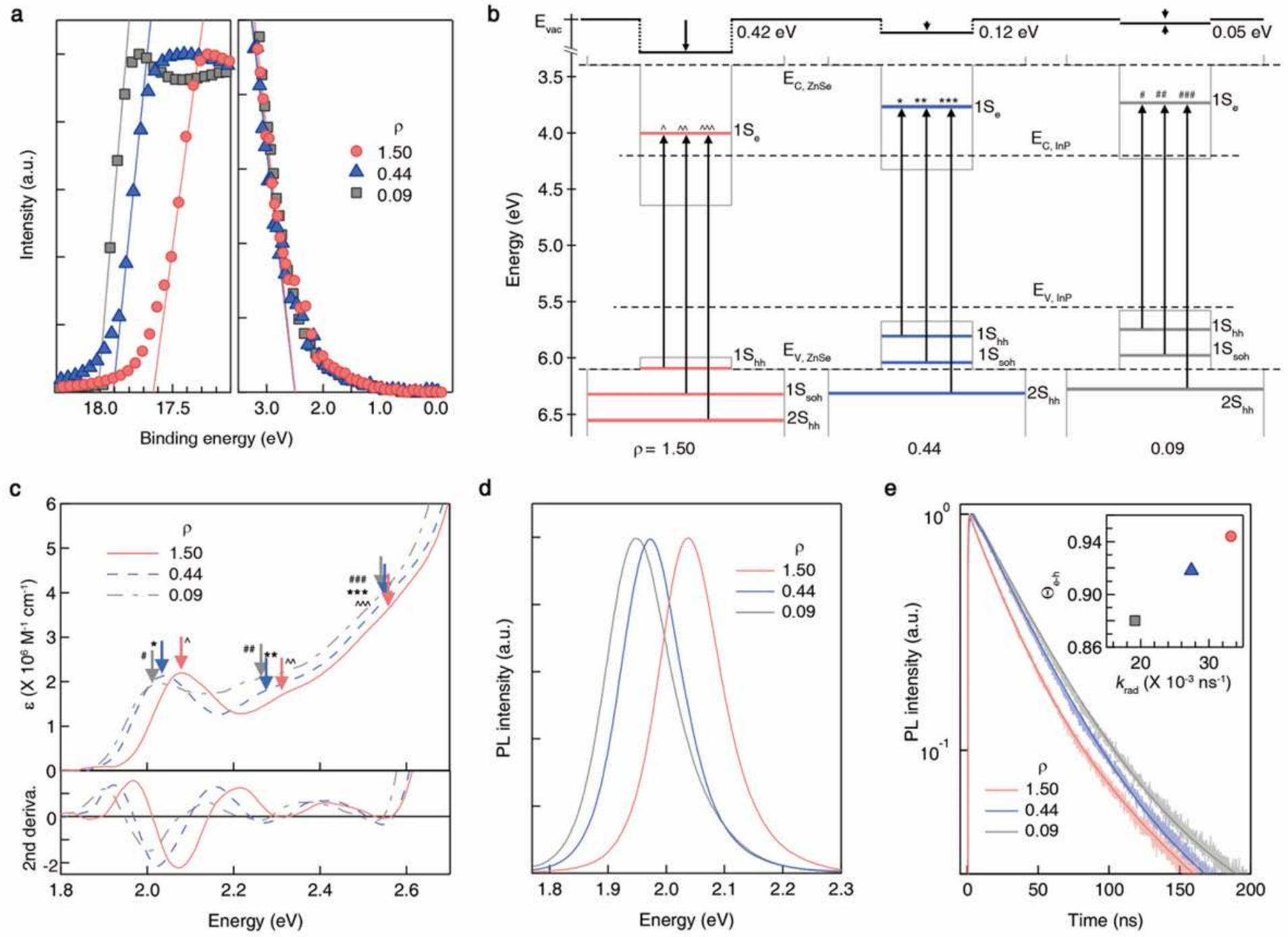

Figure 2

Electronic and photophysical characteristics of InP/ZnSe h-NCs for various In:P ratios (i.e., for varying interfacial dipole densities, $\rho$ ). (a) Ultraviolet photoemission spectra at the high-binding-energy (left) and the low-binding-energy cutoff region (right) of $\operatorname{InP}(r=1.9 \mathrm{~nm}) / \mathrm{ZnSe}(\mathrm{H}=2.2 \mathrm{~nm}) \mathrm{h}-\mathrm{NCs}$ with $\rho=1.50$ (red), 0.44 (blue) and 0.09 (gray) $n m-2$. (b) The potential profile and the electronic energy levels in $\ln P(r=$ $1.9 \mathrm{~nm}) / \mathrm{ZnSe}(\mathrm{H}=2.2 \mathrm{~nm}$ ) h-NCs with $\rho=1.50$ (left), 0.44 (middle) and 0.09 (right) nm-2. Dashed lines denote bulk band edge positions of ZB InP and ZB ZnSe. Dim solid lines indicate the band edge profile of $\mathrm{InP} / \mathrm{ZnSe}$ h-NCs with the interfacial polarization. The lowest quantized state of conduction band (1Se) and the quantized states of valence band (1Shh, 1Ssoh, and 2Shh) of h-NCs are noted with colored solid lines. Optical transitions (1Shh-1Se, 1Ssoh-1Se, and 2Shh-1Se) at each h-NC are indicated with arrows and labels. (c) Absorbance spectra (top) and their 2nd derivatives (bottom), (d) photoluminescence spectra, and (e) single exciton decay dynamics (inset: electron-hole wavefunction overlap integral (Oe-h) versus radiative recombination rate $(\mathrm{krad}))$ of $\mathrm{InP}(\mathrm{r}=1.9 \mathrm{~nm}) / \mathrm{ZnSe}(\mathrm{H}=2.2 \mathrm{~nm})$ h-NCs with $\rho=1.50$ (red), 0.44 (blue) and 0.09 (gray) nm-2. 
a
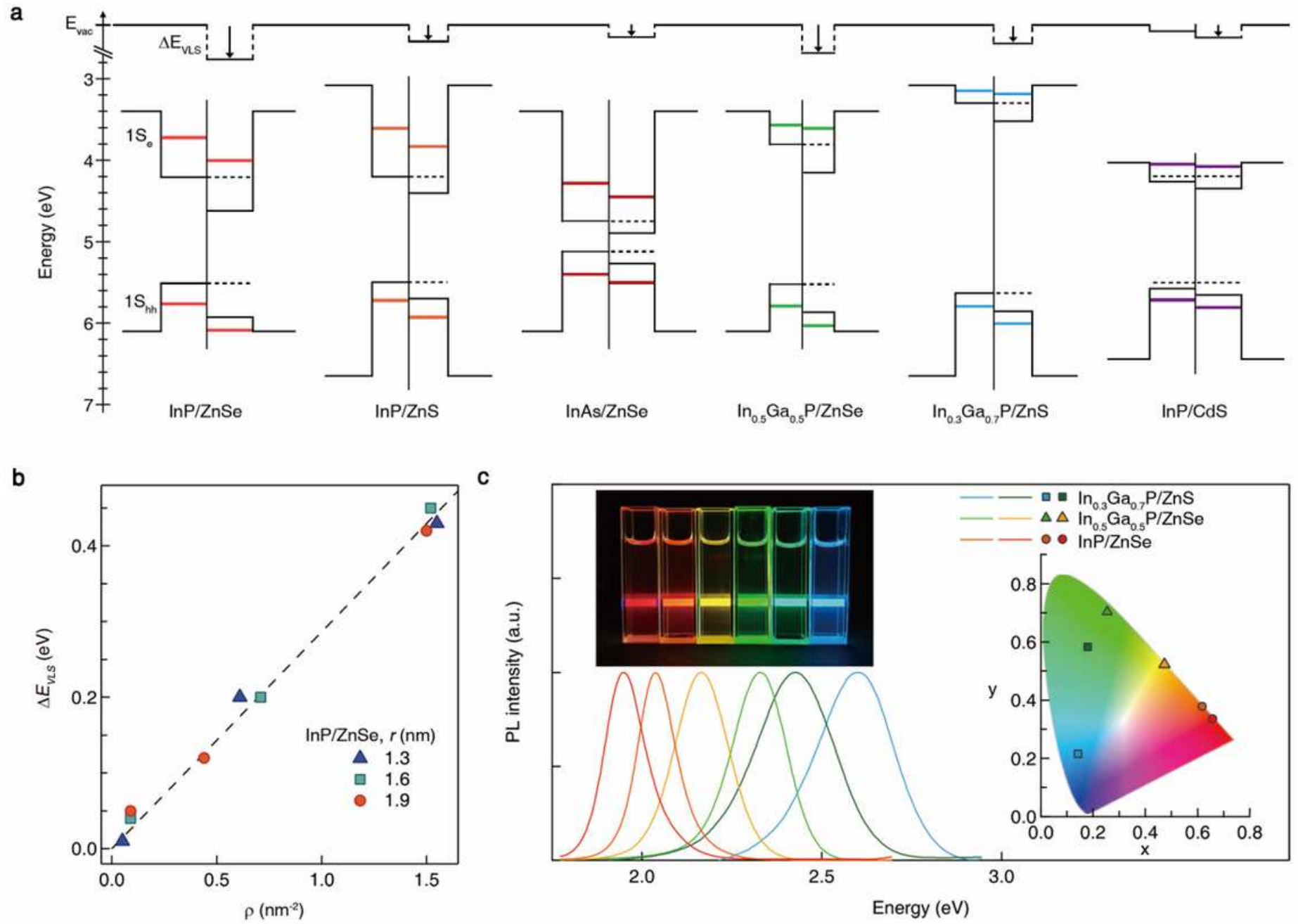

Figure 3

Universal polarization effect at the interface on electronic and optical characteristics of III-V/II-VI h-NCs.

(a) Experimentally gained vacuum-level shift ( $\triangle E V L S)$ in InP/ZnSe, InP/ZnS, InAs/ZnSe,

In $0.5 \mathrm{Ga0} .5 \mathrm{P} / \mathrm{ZnSe}$, In0.3Ga0.7P/ZnS, and InP/CdS h-NCs upon the change of the interfacial polarization. Top solid line indicates the vacuum level (Evac). The vertical arrows denote experimentally achievable vacuum-level shift with the interfacial polarization for each h-NCs. Left and right halves for each h-NCs contrast the potential profile of h-NCs with the least and highest dipole densities among experimentally achieved values, respectively. The colored solid lines indicate the lowest quantized states of conduction band (1Se) and valence band (1Shh) for each case. (b) The extent of vacuum-level shift ( $\triangle E V L S)$ versus interfacial dipole density $(\rho)$ in InP/ZnSe h-NCs of varying $\ln P$ radii $(r=1.3$ (blue), 1.6 (green), and 1.9 (red) $\mathrm{nm}$ ). The ZnSe thickness $(\mathrm{H})$ is fixed to be $2.2 \mathrm{~nm}$. The dotted line is a linear fit to the data. (c) Achievable photoluminescence spectra from $\ln 0.3 \mathrm{Ga0} .7 \mathrm{P}(\mathrm{r}=1.2 \mathrm{~nm}) / \mathrm{ZnS}(\mathrm{H}=2.6 \mathrm{~nm}), \ln 0.5 \mathrm{Ga} 0.5 \mathrm{P}(\mathrm{r}=$ $1.3 \mathrm{~nm}) / \mathrm{ZnSe}(\mathrm{H}=3.2 \mathrm{~nm})$ and $\operatorname{lnP}(\mathrm{r}=1.9 \mathrm{~nm}) / \mathrm{ZnSe}(\mathrm{H}=2.2 \mathrm{~nm}) \mathrm{h}-\mathrm{NCs}$ by varying the interfacial dipole densities. Insets are a photograph of resulting h-NCs and their color space in the CIE 1931 chromaticity diagram. 
a

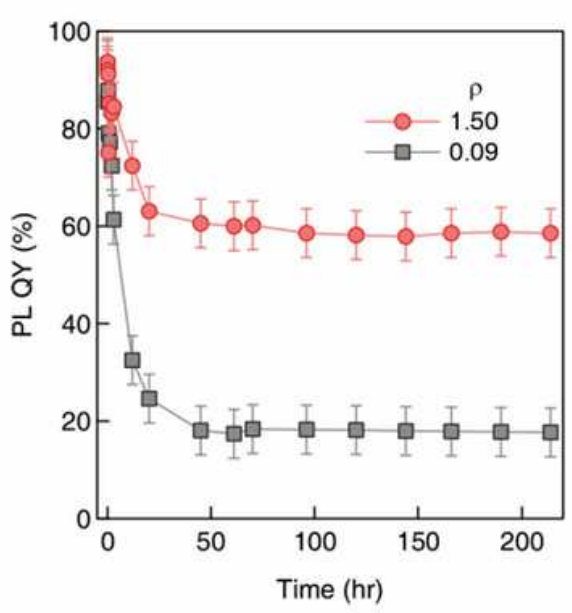

b

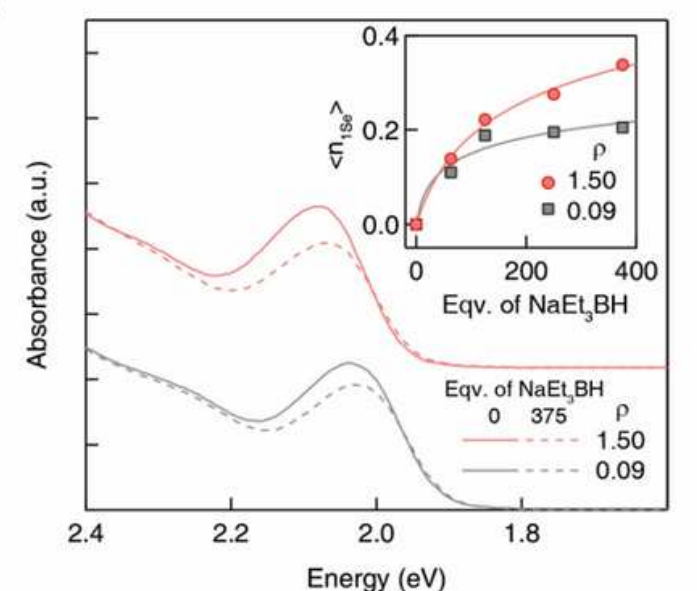

c

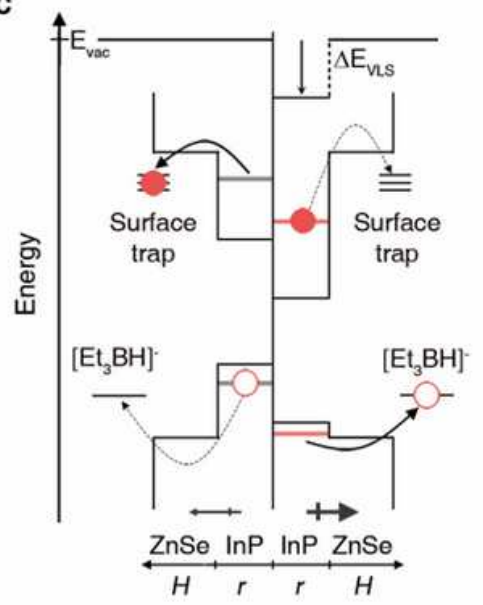

d

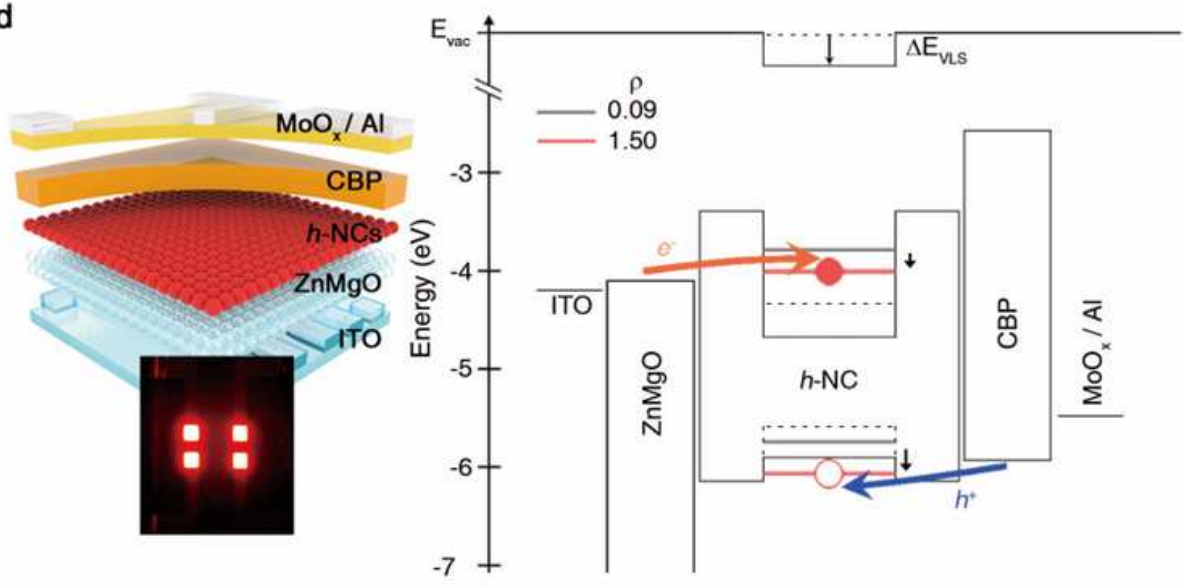

e

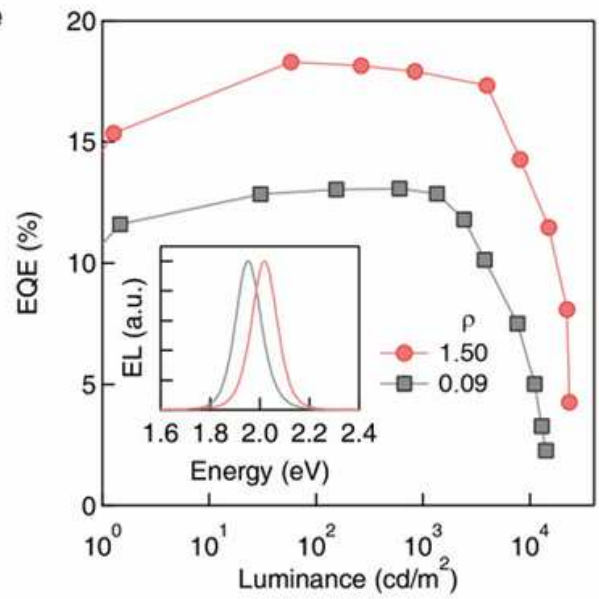

Figure 4

Impact of the interfacial polarization on transport characteristics of charge carriers across InP/ZnSe heterovalent h-NCs. (a) Temporal change in PL QYs of InP/ZnSe h-NCs with $\rho=1.50$ (red) versus 0.09 (gray) nm-2 under exposure to air. The average (symbols) and standard deviation (error bars) were calculated from multiple independent runs of the experiment. (b) Absorption spectra of InP/ZnSe h-NCs with $\rho=1.50$ (red) versus 0.09 (gray) $\mathrm{nm}-2$ without (solid lines) and with the addition of 375 equivalents of sodium triethylborohydride $(\mathrm{NaEt} 3 \mathrm{BH})$ (dotted line). The absorption spectra are vertically shifted for visual clarity. Inset shows the electron occupancy at 1 Se as a function of the amount of reductant. (c) Schematic contrasting the injection and ejection characteristics of charge carriers in h-NCs with $\rho=1.50$ (left) versus 0.09 (right) nm-2. (d) Schematic illustration and photograph (left) and the energy band diagram of NC-LED employing InP/ZnSe h-NCs (right). The potential profile and electronic states (1Se and 1Shh) of InP/ZnSe h-NCs with $\rho=1.50$ (red) versus 0.09 (gray) nm-2 are overlayed for comparison. (e) The external quantum efficiency (EQE) versus luminance curves of NC-LEDs employing InP/ZnSe hNCs with $\rho=1.50$ (red) versus 0.09 (gray) $\mathrm{nm}-2$. Inset is the electroluminescence spectra of NC-LEDs employing InP/ZnSe h-NCs with $\rho=1.50$ (red) versus 0.09 (gray) nm-2.

\section{Supplementary Files}


This is a list of supplementary files associated with this preprint. Click to download.

- SIHeterovalentNCNatureMaterials.docx 